\title{
The Investigation of the Effect of Laser Radiation on the Dielectric Properties of Polymethylmethacrylate
}

\author{
Torshkhoyeva Z. S. ${ }^{1, *}$, Akhriev A. S. ${ }^{2}$, Kunizhev B. I. ${ }^{3}$ \\ ${ }^{1}$ Department of General Physics, Ingush State University, Russia \\ ${ }^{2}$ Theoretical Physics Department, Ingush State University, Russia \\ ${ }^{3}$ Theoretical Physics Department, Kabardino-Balkarian State University, Russia
}

Copyright $\mathrm{O} 2016$ by authors, all rights reserved. Authors agree that this article remains permanently open access under the terms of the Creative Commons Attribution License 4.0 International License

\begin{abstract}
This article examined the impact of laser radiation on the dielectric properties of polymethylmethacrylate at frequency of $50 \mathrm{kHz}$ within the temperature interval from 170 to $420 \mathrm{~K}$. The authors showed that the processing of the samples with laser radiation leads to substantial changes in the dielectric constant and tangent of dielectric loss angle.
\end{abstract}

Keywords Polymethylmethacrylate (PMMA), the Destruction, Crack, the Laser Pulse

\section{Introduction}

The given paper presents us the results of the study of the dielectric relaxation processes in polymethylmethacrylate under laser irradiation at frequency of $50 \mathrm{kHz}$ within the temperature interval from 170 to $420 \mathrm{~K}$.

The problem of studying the interaction of laser radiation with polymeric materials lies in the field of studying the impact of intense energy flows on the substance. The effect of laser radiation on polymer materials was studied according to the changes in the dielectric parameters of irradiated polymethylmethacrylate. The changes in the dielectric permittivity $\varepsilon$ 'and the tangent of dielectric loss angle $\operatorname{tg} \delta$ depending on the intensity of the laser radiation, the frequency of the external field, temperature and time were studied by the method of dielectric loss.

The samples were exposed to laser radiation with a wavelength of $\sim 511 \mathrm{~nm}$. Laser pulses having duration of about $25 \mathrm{~ns}$ and following with frequency of $8.0 \mathrm{kHz}$, irradiated polymer surface within $5 \mathrm{~s}$. The laser power reached 4.0 watts. The irradiated spot size of $2.5 \mathrm{~cm}^{2}$ illuminated the sample with area of $8 \mathrm{~cm}^{2}$ by means of scanning. The maximum intensity of the radiation in the pulse at the sample surface varied from 3.0 to $9 \mathrm{~kW} / \mathrm{cm}^{2}$.

The powers of the laser irradiation, achieved in our experiments, contributed to heat of the optically transparent medium of PMMA. In this case the temperature of polymethylmethacrylate softening was not achieved.

Despite the fact that the intensity of the laser radiation in our experiments reached its maximum value $\sim 9 \mathrm{~kW} / \mathrm{cm}^{2}$ laser irradiation didn't reach the damage threshold that was observed visually, as the investigated sample of PMMA was transparent and wavelength $511 \mathrm{~nm}$ was in the optical range. But such impact can cause hidden changes. As it turned out, the dielectric parameters of the investigated samples vary significantly due to the laser irradiation.

Figure 1 shows the temperature dependence of the dielectric permittivity at frequency of $50 \mathrm{kHz}$ : initial - curve 1 , after 30 hours of exposure - line 2 and after two months of exposure - curve 3 .

The figure shows that after 30 hours of irradiation the permittivity of PMMA increases 1.2-1.5 times in the temperature range from 170 to $270 \mathrm{~K}$. On further temperature increasing the dielectric permittivity of the irradiated PMMA increases linearly and reaches a three-fold increase as compared to the value $\varepsilon^{\prime}$ of the initial PMMA at the given temperature (curve - 2). 


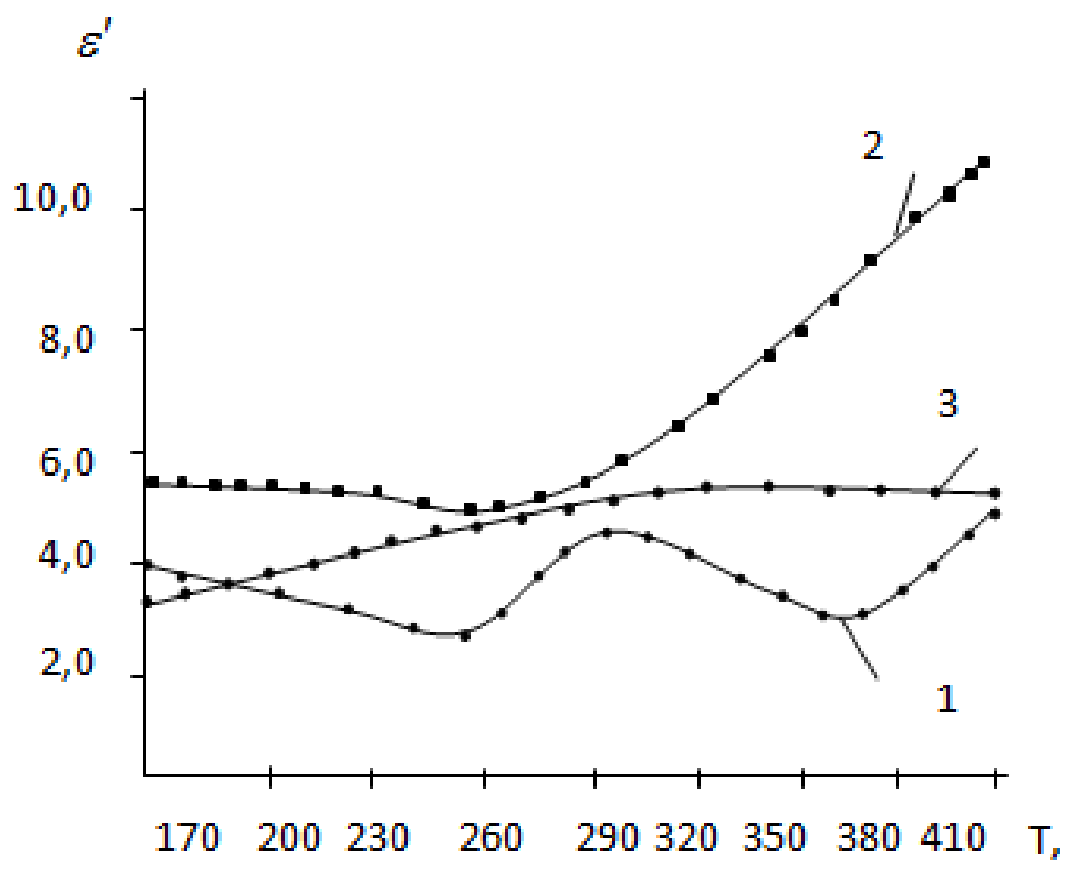

Figure 1. The dependence on T for PMMA at frequency of 50kHz.1 -- initial , $2-30$ hours, $3-2$ months after irradiation. Iimp $=5 \mathrm{~kW} / \mathrm{cm}^{2}$.

After two months of laser irradiation the dielectric permittivity of irradiated samples of polymethylmethacrylate at low temperatures of 170 to $200 \mathrm{~K}$ reduces to $\varepsilon^{\prime} \sim 4$ of the original non-irradiated PMMA under the influence of relaxation processes (curve 3, Figure 1). The dielectric permittivity has $\varepsilon^{\prime} \sim 5,0$ in the temperature range from 200 to $410 \mathrm{~K}$ that is $30 \% \varepsilon^{\prime}$ higher than the original sample. This value corresponds to a new equilibrium of PMMA.

The temperature dependences of the tangent of dielectric loss angle from temperature for PMMA at frequency of 50 $\mathrm{kHz}$ are shown in Figure 2 (initial - curve 1, after 30 hours of irradiation - curve 2 and after two months - curve 3), J of impulse $=5 \mathrm{~kW} / \mathrm{cm}^{2}$.

The graph shows that the laser irradiation leads to increasing in the dielectric loss of PMMA at temperatures ranging from 170 to $440 \mathrm{~K}$ (curve 2). In this case the first two maxima disappear, merge together, and $\alpha$ - relaxation process becomes apparent in the same temperature range as it does for the initial non-irradiated sample of polymethylmethacrylate. Two months after the exposure $\operatorname{tg} \delta$ slightly reduces as compared to tg $\delta$ samples measured in 30 hours after irradiation. They do not relax to $\operatorname{tg} \delta$, corresponding to non-irradiated samples of PMMA. In addition, it does not show $\beta$-max, and $\alpha$-maxima shift to $15-20 \mathrm{~K}$ lower temperatures and appear as one maximum relaxation.

Thus, our studies have shown that the treatment of the samples by a laser pulse leads to significant changes in the dielectric permittivity $\varepsilon$ 'and the tangent of dielectric loss angle tg, measured in a wide temperature range at frequency of $50 \mathrm{kHz}$.

Consider the causes of emerging the irreversible changes in the dielectric parameters of the PMMA samples treated by laser radiation [1]. If the macromolecular chains breaks occur as a result of laser irradiation the dielectric losses $\operatorname{tg} \delta$ and the dielectric permittivity $\varepsilon$ ' can increase. But if a powerful laser treatment leads to the emergence of new cross-links in the PMMA macromolecules, $\varepsilon$ 'and tg $\delta$ may reduce. The emerging of macro-chains rupture can be caused by thermal fluctuation and photodissociation mechanisms; they may have strain origin associated with temperature gradients within the area of the laser pulse and the occurrence of electromagnetic stress in the PMMA samples due to unevenness of thermal expansion, and with excitation dipole groups relaxation processes of laser pulses exposure close in the action time to the dipole relaxation time groups. 


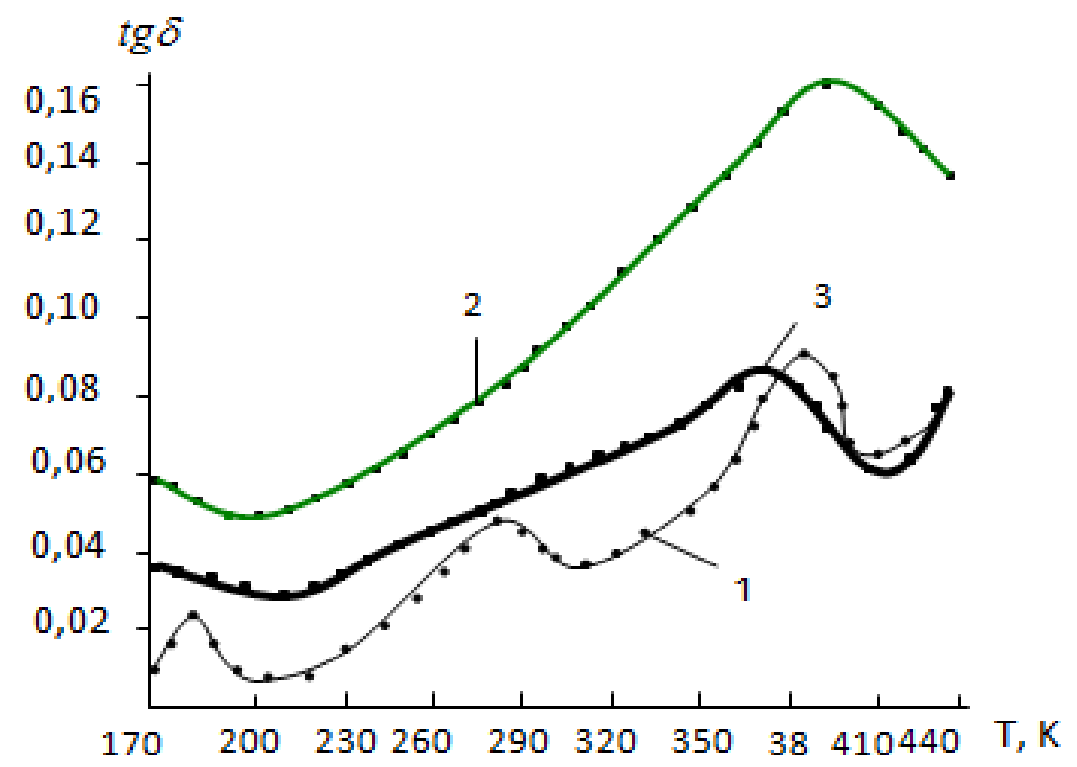

Figure 2. The dependence of the tangent dielectric loss angle of temperature for PMMA at frequency of 50kHz. 1 - initial, 2 - 30 hours, 3 - 2 months after irradiation. Iimp и ч. $\mathrm{I}=5 \mathrm{~kW} / \mathrm{cm}^{2}$

By the above given values of dielectric parameters of the PMMA samples subjected to laser irradiation and corresponding to the new equilibrium of PMMA (removed two months after irradiation) and significantly differing from the corresponding properties of the original PMMA can cause a variety of laser energy absorption mechanisms by means of transparent solids. They can be their own arrangements associated with the properties of the polymer matrix (impact and multiphoton ionization), and mechanisms caused by absorbing inclusions, namely thermoelastic, photoionization of thermal explosion.

Changes in physical properties arising under the action of laser radiation in transparent polymers can be divided into two groups. The first group includes the changes that occur in a perfectly clean environment, and the second one includes the changes due to impurities. The experimentally discovered non-returning values $\varepsilon$ 'and tg $\delta$ of PMMA to the initial values of $\varepsilon^{\prime}$ and $\operatorname{tg} \delta$ in the entire temperature and frequency range, are connected with the improper absorption mechanisms of the laser radiation in the used field of nanosecond range of laser exposure. This explanation of the found facts is in good agreement with the data of Manenkov A. A. and Nechitailo V. S. [2]. We shouldn't rule out the influence of relaxation processes with relaxation times that are close to the values of the laser action duration.

There is the intense absorption of laser radiation in the places of micropores, microcracks and foreign impurities location. This leads to overheating of polymethylmethacrylate in these places Microcracks and micropores can be filled partially or completely with gases of different chemical compositions, depending on the laser energy exposure.

Work [3] shows that, the data of chemical composition of the gas filling the inner cavity of the cracks formed during the laser destruction of polymethylmethacrylate is shown by laser irradiation of PMMA in the nanosecond range and facilities, close to the conditions of our experiments. It turned out that the gas composition doesn't depend on the wavelength and intensity of the laser pulse. The heavier hydrocarbons of limited (C2H6, C3H8, C4H10) and unlimited rows $(\mathrm{C} 2 \mathrm{H} 4, \mathrm{C} 3 \mathrm{H} 6)$ are found in laser pyrolysis products of PMMA.

\section{Conclusions}

As a result of the study of the temperature dependences of the dielectric permittivity and tangent of dielectric loss angle of PMMA exposed to nanosecond laser pulses, it was found that after two months laser irradiation the dielectric permittivity of the PMMA samples reduces under the influence of relaxation processes at temperatures ranging from 170 to $200 \mathrm{~K}$ to values of $\varepsilon$ ' $\sim 4$ of initial non-irradiated PMMA, and in the temperature range from 200 to $420 \mathrm{~K}$ the dielectric permittivity value is $\varepsilon^{\prime} \sim 5,0$ that is $30 \% \varepsilon^{\prime}$ ' higher than the initial non-irradiated sample. $\operatorname{Tg} \delta$ do not also relax to $\operatorname{tg} \delta$, corresponding to the original samples of PMMA.

\section{REFERENCES}

[1] Savintsev A. P. Temrokov A. I. Exposure to repetitively pulsed laser radiation on PVC .//Thermophysics of High Temperature. 2002. V. 40. №4. pp. 559-562.

[2] Manenkov A. A. Nechitailo V. S. // Quantum Electronics. 1980. V. 5. pp. 616-619.

[3] Butenin A. V., Kogan B. D. // Quantum Electronics. 1986. V. 13. pp. 2149-2151. 